Revisión

\title{
Abordaje terapéutico del carcinoma de pulmón no microcítico avanzado: ¿estamos "avanzando"?
}

D. Isla, R. Lastra, E. Aguirre, E. Ortega, J. Lambea, R. Andrés

\section{Resumen}

La quimioterapia en cáncer de pulmón no microcítico avanzado consigue un pequeño pero consistente beneficio así como paliación de síntomas relacionados con el cáncer. En la actualidad, el tratamiento en $1^{\mathrm{a}}$ línea se basa en dobletes con fármacos de $3^{\mathrm{a}}$ generación con o sin platino.

Las terapias dirigidas se muestran prometedoras por su actividad y tolerancia. La aplicación de la genómica es hoy también una vía de investigación.

Actualmente, se pueden considerar segundas, terceras o más líneas de tratamiento, y también una administración individualizada para performance status 2 o ancianos. Existen diferencias relacionadas con el sexo, desde su causa a los resultados.

Ciertamente, estamos avanzando en el tratamiento del cáncer de pulmón no microcítico, aunque lentamente. La investigación de fármacos más eficaces, la selección de pacientes basada en factores clínicos y la biología del tumor, y la óptima combinación de las diferentes modalidades terapéuticas, son la prioridad de los ensayos del futuro.

\section{Palabras clave:}

Cáncer de pulmón no microcítico avanzado. Tratamiento. Avances.

Oncología, 2005; 28 (7):311-320 


\section{Summary}

Chemotherapy has a place in the treatment of advanced non-small cell lung cancer achieving a palliation of the disease-related symptoms and a small but consistent survival benefit. At present, the first line treatment is based on third generation drugs doublets with or without platinum.

Targeted therapies seem promising because of their activity and tolerance. Application of genomics to nonsmall cell lung cancer therapy represents also an interesting research way for personalized treatments.

Second, third and more lines of treatment have to be considered, as well as adequate individualized treatments for performance status 2 and elderly patients. Sex-related differences have been determined in causation and results.

Advances in the treatment of non-small cell lung cancer have certainly been achieved, although slowly. Investigation of more efficient drugs, selection of patients based on clinical factors and tumor biology, and the optimal way to combine the different therapeutic modalities, are to be the priority for future trials.

Key words: Advanced non-small cell lung cancer. Treatment. Advances.

\section{Tratamiento en $1^{\text {a }}$ línea}

El meta-análisis de $1995^{1}$ muestra un aumento de supervivencia con combinaciones basadas en cisplatino $(\mathrm{HR}=0.73)$ y con mejora en la calidad de vida. En esta última década se han incorporado nuevos agentes (taxanos, vinorelbina, gemcitabina) que se han combinado con platino en esquemas superiores a cisplatino, a estos agentes en monoquimioterapia (meta-análisis) ${ }^{2}$ o a regímenes más clásicos, en términos de supervivencia y respuesta, convirtiéndose en el tratamiento standard ${ }^{3,4}$. El meta-análisis de monoquimioterapia vs dobletes determina mejores resultados para los dobletes ${ }^{5}$, aunque en algún estudio no sea así (paclitaxel vs paclitaxel/ carboplatino $)^{6}$. Estos dobletes de nuevos agentes con platino han sido comparados entre si observándose equivalencia entre ellos (respuestas de 20-35\% y supervivencia mediana 7-11 m.) con diferente perfil de toxicidad (estudios ECOG 1594, TAX 326, ILCP, SWOG 9509) ${ }^{7-10}$. Por tanto, la decisión terapéutica desde el punto de vista clínico será individualizada considerando también otros factores como toxicidad, comorbilidades, conveniencia paciente/médico, costes...

La duración de la QT no debe exceder de 4 ciclos si existe estabilización de la enfermedad, y no más de 6 ciclos si hay respuesta ${ }^{3}$ (no hay evidencia de mejor supervivencia hasta progresión), pues un número mayor sólo conduce a un aumento de toxicidad con deterioro de calidad de vida ${ }^{11}$.

El meta-análisis de gemcitabina/platino vs nogemcitabina/platino ha demostrado una mejor supervivencia y tiempo a la progresión para gemcitabina/platino (beneficio absoluto de tiempo a la progresión de $4.2 \%$ y de supervivencia global de $3.9 \%)^{12}$.

Respecto a la superioridad de cisplatino vs carboplatino, hay estudios en un sentido y en otro ${ }^{13,14}$, pero un reciente meta-análisis determina una diferencia del $11 \%$ de supervivencia superior para cisplatino con un nuevo agente $(\mathrm{P}=0.039)^{15}$ (Fig. 1).

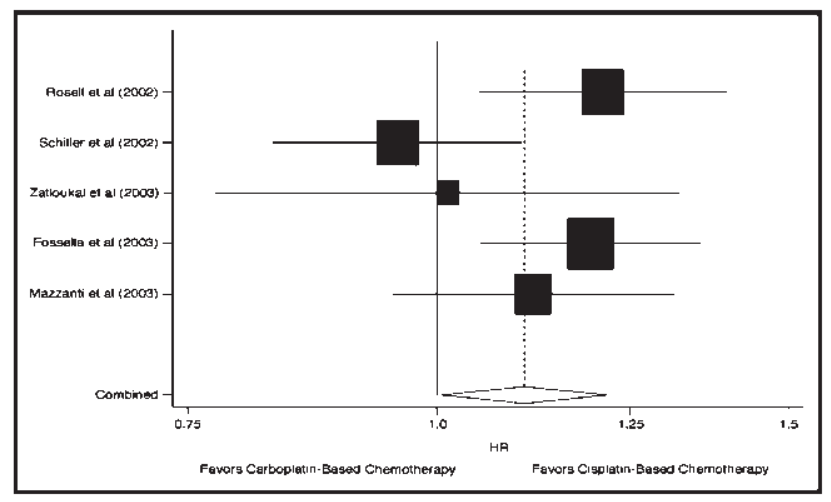

Figura 1. Meta-análisis de combinaciones con cisplatino vs carboplatino. 


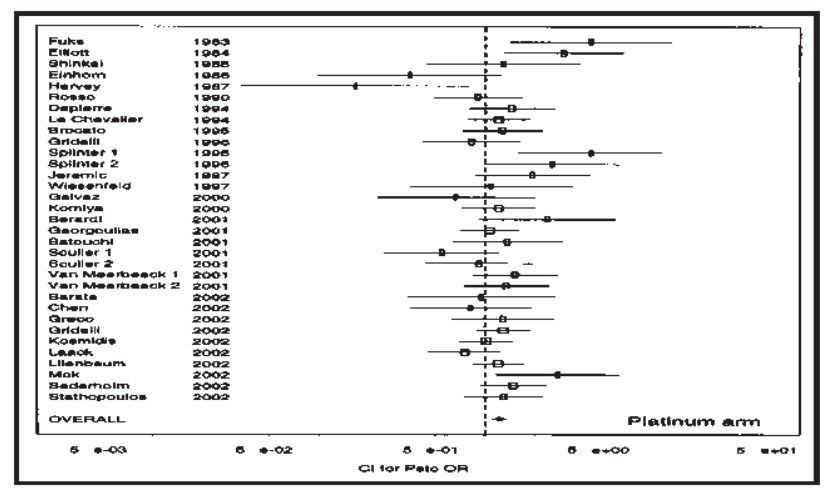

Figura 2. Meta-análisis de combinaciones con o sin platino: Todos los estudios.

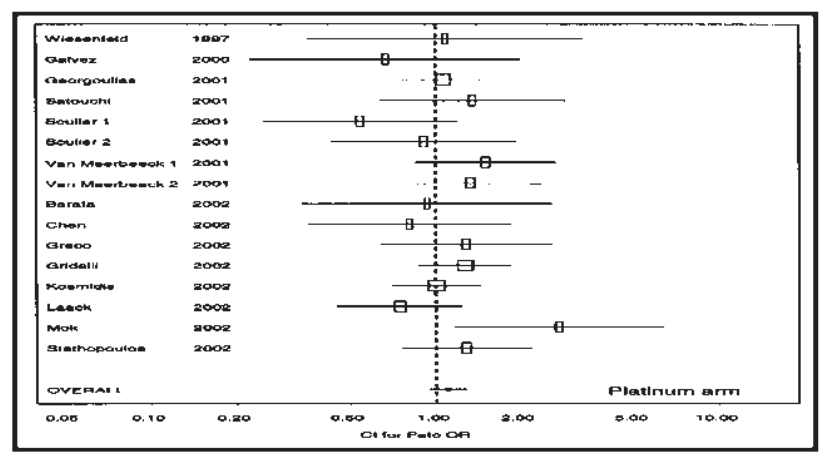

Figura 3. Meta-análisis de combinaciones con o sin platino: Estudios sin platino con fármacos de $3^{\mathrm{a}}$ generación

Las combinaciones sin platino han sido valoradas frente a con platino en un reciente meta-análisis demostrándose que son menos activas en cuanto a respuesta (aspecto importante si se emplean como terapias de inducción en tratamientos con intención curativa) con un 5\% menos de supervivencia a 1 año, pero sin diferencias en supervivencia cuando se consideran sólo las combinaciones sin platino con fármacos de $3^{\text {a }}$ generación $(\mathrm{P}=.17)$. La toxicidad es mayor para combinaciones con platino. La conclusión sería que los dobletes de $3^{a}$ generación sin platino son una opción alternativa ${ }^{16}$ (Figs. 2 y 3 ).

Los tripletes no han demostrado superioridad respecto de los dobletes (mayor respuesta pero no supervivencia) aunque si mayor toxicidad ${ }^{5,17}$.

La estrategia de tratamiento de mantenimiento tras no progresión a la quimioterapia está siendo de nuevo considerada en diferentes estudios. Paclitaxel semanal tras quimioterapia con Carbo/PAC no ha dado resultados en un estudio fase III $^{18}$. Otros estudios se encuentran en marcha: Estudio B9E-USS245 en el que se administra GEM/Carbo x 4 ciclos y luego se randomiza a docetaxel inmediatamente o a la progresión (resultados muy preliminares presentados en el Congreso de ASCO-2005 ${ }^{19}$ ), Estudio JMEN randomizado en el que tras quimioterapia se administra Pemetrexed vs mejor cuidado de soporte, y el Estudio EORTC 08021 que tras quimioterapia randomiza a gefitinib vs placebo.

En cuanto a las terapias dirigidas, hay que destacar los recientes hallazgos sobre mutaciones del gen EGFR que se presentan sobre todo en adenocarcinomas, no fumadores, mujeres y asiáticos (10-20\% en población caucasiana $)^{20,21}$. Cuando estas existen, se consiguen con los inhibidores EGFR-TK respuestas muy satisfactorias y larga supervivencia. Parece necesario realizar una selección clínica y molecular para el uso de inhibidores EGFR-TK los cuales no han demostrado beneficio en estudios fase III cuando se asocian a combinaciones de quimioterapia sin contemplar dicha selección (INTACT 1 y 2, TRIBUTE, TALENT) ${ }^{22-25}$. Las mutaciones EGFR son el mejor factor predictivo en estos momentos para el tratamiento con inhibidores EGFR-TK, pero este no es del todo perfecto porque hay respuestas sin mutaciones y mutaciones sin respuestas. Disponemos recientemente de diferentes resultados en el análisis retrospectivo de mutaciones de estudios conocidos (TIBUTE, TALENT, INTACT, IDEAL, S0126, BR21) en el Congreso de ASCO 2005, y de otros resultados de estudios publicados en los que en algunos también se definen otros marcadores predictivos de eficacia: FISH/IHQ de EGFR, IHQ de pAKT, mutaciones K-ras ${ }^{26-30}$ (Tabla I). Además, probablemente las mutaciones EGFR sean un factor pronóstico, demostrado en los estudios TRIBUTE e INTACT (mejor supervivencia en pacientes mutados que recibieron o no inhibidores EGFR-TK), y porque los resultados de supervivencia en pacientes sin mutaciones que responden son menos satisfactorios.

Por otro lado, han sido detectadas mutaciones adquiridas en EGFR (exón 20) en pacientes resistentes a inhibidores EGFR-TK, aunque seguramente existan también a otros niveles. Se valoraría entonces la posibilidad de administración de $2^{a}$ línea de tratamiento con inhibidores EGFR.

Necesitamos llevar a cabo estudios prospectivos 
TABLA I

Estudios con inh. EGFR y determinación de mutaciones EGFR

\begin{tabular}{|c|c|c|c|c|c|}
\hline Estudio & Farmaco & Mutaciones & $\begin{array}{l}\text { Respuesta } \\
\text { si Mutaciones }\end{array}$ & $\begin{array}{c}\text { Supervivencia } \\
\text { med. si Mutaciones }(m .)\end{array}$ & Diferencias \\
\hline TRIBUTE & Erlotinib & $12.7 \%$ & $53 \%$ & $?$ & Mejor (NS) \\
\hline TALENT & Erlotinib & & & $?$ & NS \\
\hline INTACT & Gefitinib & $10.2 \%$ & $72 \%$ & $?$ & Mejor? \\
\hline IDEAL & Gefitinib & $18 \%$ & $46 \%$ & $?$ & ? \\
\hline S0126 & Erlotinib & $18 \%$ & $23 \%$ & $?$ & $?$ \\
\hline BR21 & Erlotinib & $23 \%$ & $15 \%$ & $?$ & NS \\
\hline $\operatorname{Han}^{26}$ & Gefitinib & $18.9 \%$ & $64 \%$ & 30 & $\mathrm{P}<0.001$ \\
\hline Cappuzzo $^{27}$ & Gefitinib & $17 \%$ & $54 \%$ & 20 & $\mathrm{P}=0.09$ \\
\hline Mitsudomi $^{28}$ & Gefitinib & $56 \%$ & $82 \%$ & $?$ & $\mathrm{P}=0.0053$ \\
\hline Cortés-Funes ${ }^{29}$ & Gefitinib & $12 \%$ & $60 \%$ & 13 & $\mathrm{P}=0.02$ \\
\hline Tarón ${ }^{30}$ & Gefitinib & $28 \%$ & $94 \%$ & 18 & $\mathrm{P}=0.04$ \\
\hline
\end{tabular}

con inhibidores EGFR, conocemos los resultados ya de algunos ${ }^{31-32}$, y hay otros en marcha. En la actualidad, las líneas de investigación se orientan hacia el desarrollo de otros inhibidores EGFR de más amplio espectro que puedan ser activos también si no hay mutaciones o tras su resistencia: lapatinib, ZD6474, CI 1033...; y se considera la posibilidad de combinación con otras terapias dirigidas, QT...

Cetuximab ha sido ensayado en diferentes fases II combinado con dobletes, y destaca el estudio LUCAS, fase II randomizado de CDDP/VNR \pm cetuximab con resultados de eficacia satisfactorios para la asociación del Ac monoclonal anti EGFR ${ }^{33}$ (está ya en marcha el estudio fase III).

Otro Ac monoclonal es bevacizumab (anti VEGF) que ha sido también ensayado en un fase II randomizado con Carbo/PAC, con resultados de mejor respuesta y tiempo a la progresión con tendencia a mejor supervivencia pero con episodios de sangrado tumoral $^{34}$. El grupo ECOG ha llevado a cabo el fase III de Carbo/PAC \pm bevacizumab (E4599) con diferencias significativa de supervivencia, aunque se han seleccionado en este estudio pacientes con nocarcinoma escamoso, sin historia de HTA ni hemoptisis severa, y la toxicidad es superior con mejores resultados en varones. Es el primer estudio fase III de quimioterapia asociada a una terapia dirigida que demuestra mejores resultados para la combinación (supervivencia mediana $12.5 \mathrm{~m}$. vs $10.2 \mathrm{~m}$, $\mathrm{P}<0.007)^{35}$. Sin embargo, de momento sólo disponemos de este estudio, en pacientes muy seleccionados y con mayor toxicidad, por lo que es difícil considerar este tratamiento todavía como standard. Se encuentra en marcha el estudio fase III BO1770 con $\mathrm{CDDP} / \mathrm{GEM} \pm$ bevacizumab.

Para pacientes con Her-2/neu 3+, trastuzumab consigue buenos resultados asociado a dobletes con platino en algún estudio ${ }^{36}$.

Han sido negativos los estudios fase III con el retinoide bexaroteno combinados con Carbo/PAC y CDDP/VNR respectivamente (SPIRIT I y II) ${ }^{37,38}$.

Son de interés las investigaciones de biología molecular orientadas a seleccionar a los pacientes que vayan a responder a las diferentes terapias en relación con distintos marcadores moleculares. Entre ellas se encuentran los estudios farmacogenómicos destacando el estudio GILT del Grupo Español de Cáncer de Pulmón que es el $1^{\circ}$ en marcha que de forma prospectiva y randomizada, según niveles de ERCC1 bajos o altos a nivel del tumor (gen de reparación del DNA como marcador molecular), determina la administración de un régimen con o sin cisplatino. Se han presentado en el Congreso de ASCO 2005 resultados preliminares confirmándose una de las hipótesis de este estudio: respuesta significativamente mejor para niveles bajos de expresión de ERCC1 y quimioterapia con platino $^{39}$ (Fig. 4).

Otras líneas de investigación a nivel molecular incluyen metilaciones, sobreexpresiones de genes como TS, RRM1, BRCA1, mutaciones en diferentes genes... En este sentido, dentro del Grupo Espa- 


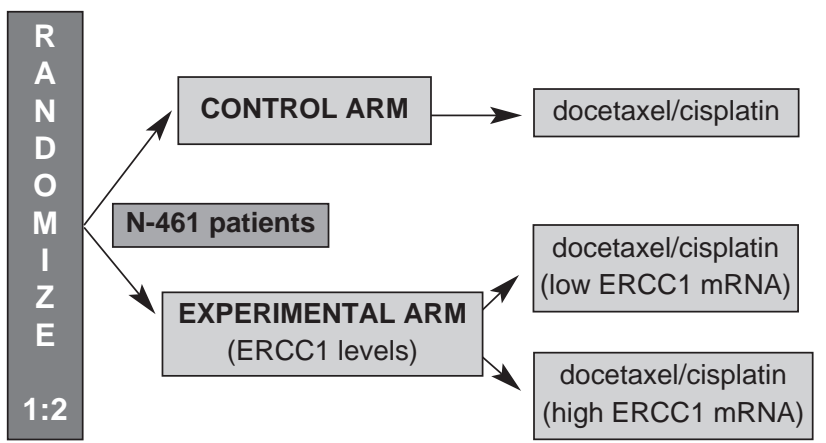

Figura 4. Estudio GILT del Grupo Español de Cáncer de Pulmón.

ñol de Cáncer de Pulmón se ha puesto en marcha el Estudio SLABD que permite determinar mutaciones EGFR en pacientes con adenocarcinoma indicando el uso de erlotinib siempre que estén presentes. Se ha creado un Banco de Tumores para valoraciones moleculares de interés en el futuro. En el Estudio SLAT se condiciona el régimen de quimioterapia a administrar en $1^{a}$ línea según los niveles de expresión de BRCA1 determinados en biopsia tumoral (Fig. 5).

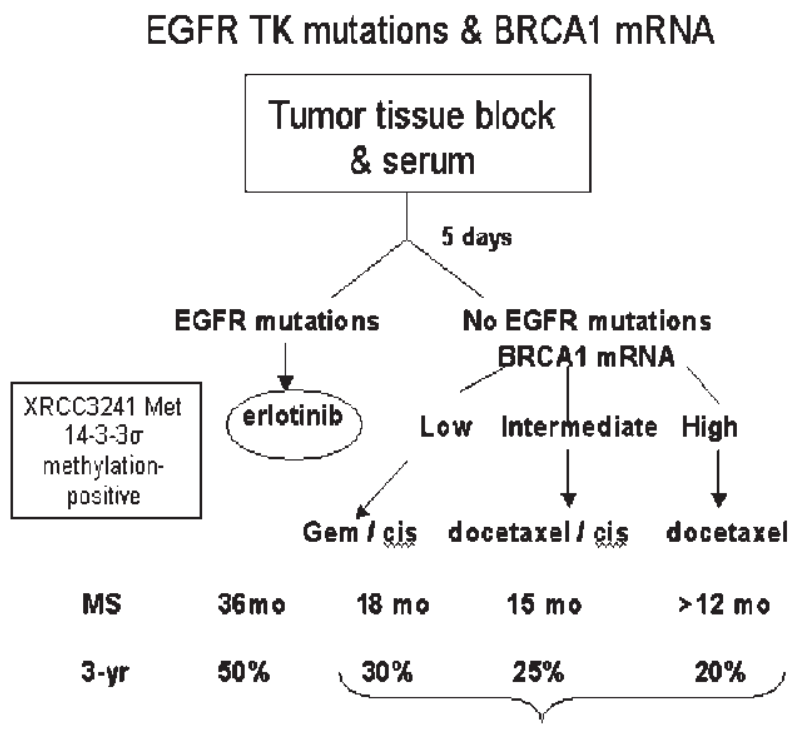

High ErbB-3 mRNA

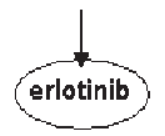

Figura 5. Estudio SLABD y SLAT del Grupo Español de Cáncer de Pulmón.

\section{Tratamiento en $2^{\mathrm{a}}$ línea}

Tras quimioterapia basada en platino y buen performance status, docetaxel se ha considerado el tratamiento standard durante los últimos años (estudios TAX 317, TAX 320) ${ }^{40-41}$. Sin embargo, posteriormente, otros fármacos han demostrado eficacia similar: pemetrexed (comparado con docetaxel en el estudio fase III JMEI, con tolerancia hematológica más favorable $)^{42}$, gemcitabina, CPT-11, dobletes sin platino...

Las recomendaciones de ESMO 2005 para la $2^{\mathrm{a}}$ línea son que docetaxel y pemetrexed mejoran los síntomas relacionados con la enfermedad y la supervivencia en pacientes seleccionados ${ }^{4}$.

Los inhibidores EGFR-TK en estudios fase II han demostrado actividad similar a docetaxel: gefitinib en estudios IDEAL 1 y $2^{43,44}$, y para erlotinib en el estudio de Pérez-Soler ${ }^{45}$, destacando la asociación de erlotinib con bevacizumab que consigue una supervivencia mediana de $12.6 \mathrm{~m}$. y a 1 año del $51.8 \%{ }^{46}$. En cuanto a estudios fase III, erlotinib es superior a cuidados de soporte en el BR. $21^{47}$; y hay otros para gefitinib: frente a cuidados de soporte $=$ estudio ISEL (no demuestra mejor supervivencia ${ }^{48}$ ), y frente a docetaxel $=$ estudio INTEREST (todavía en marcha). Gefitinib ha sido comparado con docetaxel en un estudio fase II randomizado (Estudio SIGN) demostrando misma eficacia con mejor tolerancia ${ }^{49}$. ZD6474 es un inhibidor de EGFR y VEGFR que se está valorando en la actualidad en distintos ensayos destacando los resultados frente a gefitinib (mejor tiempo a la progresión, $\mathrm{P}=0.011)^{50}$.

Bortezomib ha sido ensayado en un estudio fase II randomizado \pm docetaxel cuyos resultados son de buena tolerancia con respuesta de $9 \%$ vs $8 \%$, y supervivencia mediana 7.8 vs $7.4 \mathrm{~m}$. ( $\mathrm{P}=$ no significativa) ${ }^{51}$.

Cetuximab en monoquimioterapia consigue supervivencia a 1 año de $41 \%^{52}$. Combinado con docetaxel las respuestas alcanzan $28 \%{ }^{53}$.

\section{Performance status 2}

Recientemente se ha alcanzado un Consenso Europeo para el tratamiento de este subgrupo: se indica el uso de agentes en monoquimioterapia (gemcitabina, vinorelbina, taxanos, dependiendo la elección de 
TABLA II

Estudios Fase III en ancianos

\begin{tabular}{|lcccc|}
\hline Autor & Esquema & Respuesta Objetiva (\%) & Supervivencia med./1 a. (\%) & Diferencias \\
\hline ELVIS & VNR & 19 & $7 / 32$ & 0.05 \\
1999 & Cuid. Soporte & - & $5 / 14$ & \\
SICOG & GEM/VNR & 22 & $7 / 30$ & 0.01 \\
2000 & VNR & 15 & $4.5 / 13$ & \\
& & & $7 / 26$ & NS \\
MILES & GEM & 17 & $9 / 41$ & \\
2003 & VNR & 18 & $8 / 31$ & 0.03 \\
& GEM/VNR & 20 & $13.9 / 59.2$ & \\
WJTOG9904 & DOC & 22.7 & $9.9 / 36.5$ & \\
2005 & VNR & 9.9 & & \\
\hline
\end{tabular}

su toxicidad y la comorbilidad del paciente), aunque dobletes basados en carboplatino o dosis bajas de platino con un fármaco de $3^{a}$ generación pueden representar una alternativa ${ }^{54}$. Este Consenso coincide con las recomendaciones de ASCO 2004.

Son necesarios estudios randomizados específicos para este grupo de pacientes comparando las dos opciones ya comentadas, considerando también nuevos agentes biológicos. Además, resulta de interés la valoración de los resultados de los estudios considerando las razones que condicionan el performance status 2: comorbilidades, carga tumoral o ambas.

Es prioritario la mejora de los cuidados de soporte contemplando también como objetivos de los estudios la mejoría sintomática, el beneficio clínico y la calidad de vida.

\section{Ancianos}

En diferentes subanálisis retrospectivos de grandes estudios en los que se administraba quimioterapia basada en platino, no se han objetivado diferencias según la edad para pacientes con buenas condiciones físicas, aunque sí algo más de toxicidad. Se precisan estudios randomizados de quimioterapia basada en platino en pacientes no seleccionados.

En el Estudio ELVIS ${ }^{55}$, VNR en monoquimioterapia demostró ser superior a mejor cuidado de soporte. En el Estudio MILES ${ }^{56}$ no se demuestra que GEM/VNR sea superior a GEM ó VNR en mono- quimioterapia. Sin embargo, en el Estudio SICOG, GEM/VNR si es superior a VNR ${ }^{57}$. En el Congreso ASCO 2005 se conocen resultados del estudio fase III japonés WJTOG9904 que en $1^{\text {a }}$ línea demuestra que Docetaxel es superior a VNR (respuestas $22.7 \%$ vs $9.9 \%$, supervivencia mediana 13.9 vs $9,9 \mathrm{~m}$, $\mathrm{P}=0.03)^{58}$ (Tabla II).

Las recomendaciones del reciente Panel de Expertos Internacional para el tratamiento de ancianos $^{59}$ establece que la valoración geriátrica exhaustiva es necesaria para definir mejor el pronóstico y predecir la tolerancia al tratamiento. En pacientes no seleccionados, el tratamiento se basará en monoquimioterapia con un fármaco de $3^{\mathrm{a}}$ generación (VNR, GEM o Taxano); y para pacientes con buenas condiciones físicas con adecuada función orgánica, puede indicarse quimioterapia basada en platino. El mejor cuidado de soporte resulta especialmente importante en estos pacientes, se administre o no tratamiento de quimioterapia.

Las líneas de investigación en ancianos se orientan hacia la realización de estudios diseñados específicamente para ellos, así como a la valoración de terapias biológicas todavía no recomendadas.

\section{Mujeres y cáncer de pulmón}

La mortalidad al año por cáncer de pulmón en USA en mujeres es ya superior a la suma de muertes por cáncer de mama, útero y cérvix. 
Hay diferencias en cuanto a la presentación histológica y la edad entre hombres y mujeres: adenocarcinomas / carcinoma microcítico y edad más joven son más frecuentes en mujeres.

El riesgo de cáncer de pulmón se ha demostrado que es mayor en mujeres, con una mas alta susceptibilidad a los agentes carcinógenos del tabaco. También se han detectado diferencias moleculares entre sexos en relación con metabolismo y daño sobre el ADN producido por carcinógenos, polimorfismos (SNPs), mutaciones p53, mutaciones EGFR, mutaciones Kras...

Se ha observado la presencia de receptores estrogénicos $(\beta)$ en cáncer de pulmón no microcítico y su relación con EGFR existiendo interacción entre ambas vías. El bloqueo de los receptores de estrógenos con fulvestrant (antiestrógeno puro) y de EGFR con gefitinib determinó un incremento del efecto antiproliferativo y apoptosis en cultivos de células tumorales de pulmón y también in vivo en modelos xenógrafos ${ }^{60}$. Está en marcha un estudio fase I en mujeres post-menopáusicas con esta combinación con resultados preliminares satisfactorios ${ }^{61}$, y previsto un fase II randomizado con inhibidor EGFR con o sin fulvestrant.

Se han objetivado también diferencias en cuanto a los resultados de las terapias administradas según el sexo, pero todavía en la actualidad no se disponen de datos para poder indicar tratamientos diferentes. Si se podría ya recomendar la estratificación según este factor en los diferentes estudios, con inclusión de un $\mathrm{n}^{\circ}$ suficiente de pacientes. El grupo SWOG ha puesto en marcha el estudio S0424 que considera la determinación de marcadores moleculares relacionados con el sexo.

\section{Conclusiones finales}

En el Cáncer de Pulmón No Microcítico Avanzado el tratamiento antineoplásico aporta un pequeño aunque consistente beneficio en supervivencia así como paliación de síntomas relacionados con el cáncer. No existe un régimen de quimioterapia standard y habrá que valorar la tolerancia, conveniencia, costes, posibles mecanismos de resistencia (farmacogenómica) de las diferentes opciones terapéuticas disponibles, así como determinados factores clínicos y alteraciones moleculares, para realizar un abordaje más personalizado.
Las nuevas Terapias Dirigidas son prometedoras aunque hay mucho por conocer sobre sus posibilidades, adquiriendo gran importancia los marcadores predictivos / pronósticos relacionados.

En la actualidad, la investigación se orienta hacia la búsqueda de tratamientos antineoplásicos más eficaces, la óptima combinación de los agentes citotóxicos y biológicos, y su administración considerando la selección de pacientes a partir de la identificación de determinantes de eficacia clínicos y moleculares.

Por tanto, se puede decir que efectivamente estamos avanzando, conseguimos lentamente mejorar los resultados, pero es preciso seguir investigando de forma racional buscando el desarrollo de terapias más activas y personalizadas.

Correspondencia:

Dra. D. Isla Casado

Servicio de Oncología Médica

Hospital Clínico Lozano Blesa

C/ San Juan Bosco, 15

E-50009 Zaragoza

E-mail: onch-isla@hcu-lblesa.es 


\section{Bibliografía}

1. Non-Small Cell Lung Cancer Collaborative Group. Chemotherapy in non-small cell lung cancer: a meta-analysis using updated data on individual patients from 52 randomised clinical trials. Br Med J 1995;311:899-909.

2. Hotta K, Matsuo K, Ueoka H, et al. Addition of platinum compounds to a new agent in patients with advanced nonsmall cell lung cancer: a literature based meta-analysis of randomised trials. Ann Oncol 2004;15:1782-1789.

3. Pfister D, Johnson D, Azzoli C, et al. American Society of Clinical Oncology Treatment of Unresectable Non-small Cell Lung Cancer Guideline: Update 2003. J Clin Oncol 2004;22:330-353.

4. ESMO Minimum Clinical Recommendations for diagnosis, treatment and follow-up of non-small cell lung cancer (NSCLC). Ann Oncol 2005;16:28-29.

5. Delbaldo C, Michiels S, Syz N, et al. Benefits adding a drug to a singe-agent or a 2-agent chemotherapy regimen in advanced non-small cell lung cancer: a meta-analisys. JAMA 2004;292:470-84.

6. Lilenbaum RC, Herdon JE, List MA. Single-agent versus combination chemotherapy in advanced non-small cell lung cancer: The Cancer and Leukaemia Group B (study 9730). J Clin Oncol 2005;23:190-6.

7. Schiller J, Harrrington D, Belani C, et al. Comparison of four chemotherapy regimens for advanced non-small cell lung cancer. N Engl J Med 2002;346:92-98.

8. Fossella F, Pereira JR, von Pawel J, et al. Randomized multinational phase III study of docetaxel plus platinum combinations versus vinorelbine plus cisplatin for advanced non-small cell lung cancer: the TAX 326 study group. J Clin Oncol 2003;21:3016-24.

9. Scagliotti G, De Marinis, F, Rinaldi, M, et al. Phase III randomized trial comparing three platinum-based doublets in advanced non-small-cell lung cancer. J Clin Oncol 2002; 20:4285-91.

10. Kelly K, Crowley J, Bunn, PA Jr, et al. Randomized phase III trial of paclitaxel plus carboplatin versus vinorelbine plus cisplatin in the treatment of patients with advanced non-small-cell lung cancer: a Southwest Oncology Group Trial. J Clin Oncol 2001; 19:3210-8.

11. Socinsky MA, Schell MJ, Peterman A, et al. Phase III trial comparing a defined duration of therapy versus continuous therapy followed by second-line therapy in advanced-stage IIIB/IV non-small-cell lung cancer. J Clin Oncol 2002;20:1335-43.

12. Le Chevalier T, Scagliotti G, Natale R, et al. Efficacy of gemcitabine plus platinum chemotherapy compared with other platinum containing regimens in advanced non-small cell lung cancer. Lung Cancer 2005;47:69-80.

13. Zatloukal P, Petruzelka L, Zemanova M, et al. Gemcitabine plus cisplatin vs gemcitabine plus carboplatin in stage IIIB and IV non-small cell lung cancer: a phase III randomized trial. Lung Cancer 2003;42:321-31.

14. Rosell R, Gatzemeier U, Betticher D, et al. Phase III rando- mised trial comparing paclitaxel/carboplatin with paclita$\mathrm{xel} /$ cisplatin in patients with advanced non-small-cell lung cancer: a Cooperative Multinational Trial. Ann Oncol 2002; 13:1539-49.

15. Hotta K, Matsuo K, Ueoka H, et al. Meta-analysis of randomised clinical trials comparing cisplatin to carboplatin in patients with advanced non-small cell lung cancer. J Clin Oncol 2004;22:3860-67.

16. D’Addario G, Pintilie M, Leighl N, et al. Platinum-based versus Non-platinum-based chemotherapy in advanced non-small-cell lung cancer: a meta-análisys of the published literature. J Clin Oncol 2005;23:2926-2936

17. Alberola, V, Camps, C, Provencio M, et al. Cisplatin plus gemcitabine versus a cisplatin-based triplet versus nonplatinum sequential doublets in advanced non-small-cell lung cancer: A Spanish Lung Cancer Group Phase III Randomized Trial. J Clin Oncol 2003; 21:3207-13.

18. Belani CP, Barstis J, Perry MC, et al. Multicenter, randomised trial for stage IIIB or IV non-small-cell lung cancer using weekly paclitaxel and carboplatin followed by maintenance weekly paclitaxel or observation. J Clin Oncol 2003;21:2933-9.

19. Schiller J, Fidias P, Dakhil SR, et al. A phase III study of induction therapy with gemcitabine + carboplatin followed by either delayed vs immediate second-line therapy with docetaxel in advanced non-small cell lung cancer. Proc Am Soc Clin Oncol 2005;23:7142.

20. Lynch TJ, Bell DW, Sordella R, et al. Activating mutations in the epidermal growth factor receptor underlying responsiveness of non-small-cell lung cancer to gefitinib. N Engl J Med 2004; 350:2129-39.

21. Paez G, Janne PA, Lee JC, et al. EGFR mutations in lung cancer: correlation with clinical response to gefitinib therapy. Science 2004;304:1497-500.

22. Giaccone G, Herbst RS, Manegold C, et al. Gefitinib in Combination With Gemcitabine and Cisplatin in Advanced Non-Small-Cell Lung Cancer: A Phase III Trial-INTACT 1. J Clin Oncol 2004; 22:777-84.

23. Herbst RS, Giaccone G, Schiller JH, et al. Gefitinib in Combination With Paclitaxel and Carboplatin in Advanced Non-Small-Cell Lung Cancer: A Phase III Trial-INTACT 2. J Clin Oncol 2004; 22:785-94.

24. Gatzemeier U, Pluzanska A, Sczesna E, et al. Results of a phase III trial of erlotinib (OSI-774) combined with cisplatin and gemcitabine chemotherapy in advanced non-small cell lung cancer. Proc Am Soc Clin Oncol 2004;23:7010.

25. Herbst RS, Ptager D, Hermann, et al. TRIBUTE - A phase III trial of erlotinib (OSI-774) combined with carboplatin and paclitaxel chemotherapy in advanced non-small cell lung cancer. Proc Am Soc Clin Oncol 2004;23:7011.

26. Han S, Kim T, Hwang P, et al. Predictive and prognostic impact of epidermal growth factor receptor mutation in non-small cell lung cancer patients treated with gefitinib. J Clin Oncol 2005; 23:2493-2501.

27. Cappuzzo F, Hirsch F, Rossi E, et al. Epidermal growth factor receptor gene and protein and gefitinib sensitivity in 
non-small cell lung cancer. J Natl Cancer Inst 2005; 97:643-55.

28. Mitsudomi T, Kosaka T, Endoh H, et al. Mutations of the epidermal growth factor receptor gene predict prolonged survival after gefitinib treatment in patients with non-small cell lung cancer with postoperative recurrence. J Clin Oncol 2005;23:2513-20.

29. Cortés-Funes H, Gomez C, Rosell R, et al. Epidermal growth factor receptor activating mutations in spanish gefitinib-treated non-small cell lung cancer. Ann Oncol 2005;16:1081-6.

30. Tarón M, Ichinose Y, Rosell R, et al. Epidermal growth factor receptor (EGFR) activating mutations in the tyrosine kinase domain correlate with gefitinib response in chemorefractory non-small cell lung cancer. Proc Am Soc Clin Oncol 2005;23:7087.

31. Giaccone G, Le Chevaliere T, Smit E, et al. A phase II study of erlotinib as first-line treatment of advanced nonsmall cell lung cancer. Proc Am Soc Clin Oncol 2005;23:7073.

32. Lee DH, Han J, Lee H, et al. Gefitinib as a first-line therapy of advanced or metastatic adenocarcinoma of the lung in never-smokers. Clin Cancer Res 2005;11:3032-3037.

33. Rosell R, Daniel C, Ramlau R, et al. Randomized phase II study of cetuximab in combination with cisplatin $(\mathrm{C})$ and vinorelbine $(\mathrm{V})$ vs $\mathrm{CV}$ alone in first line treatment of patients with epidermal growth factor receptor-expressing advanced non-small cell lung cancer. Proc Am Soc Clin Oncol 2004;22:7012.

34. Johnson DH, Feherenbacher L, Novotny WF, et al. Randomized phase II trial comparing bevacizumab plus carboplatin and paclitaxel and carboplatin and paclitaxel alone in previously untreated locally advanced or metastatic nonsmall-cell lung cancer. J Clin Oncol 2004;22:2184-91.

35. Sandler A, Gray R, Brahmer J, et al. Randomized phase II/III trial of paclitaxel plus carboplatin with or without bevacizumab in patients with advanced non-squamous nonsmall cell lung cancer: An Eastern Cooperative Oncology Group (ECOG) Trial. E-4599. Proc Am Soc Clin Oncol 2005;23;LBA4.

36. Gatzemeier U, Groth G, Butts C, et al. Randomized phase II trial of gemcitabine-cisplatin with or without trastuzumab in HER2-positive non-small cell lung cancer. Ann Oncol 2004;15:19-27.

37. Blumenschein GR, Khuri F, Gatzemeir U, et al. A randomized phase III trial comparing bexarotene/carboplatin/paclitaxel versus carboplatin/paclitaxel in chemotherapy-naive patients with advanced or metastatic non-small cell lung cancer. Proc Am Soc Clin Oncol 2005;23:7001.

38. Jassem J, Zatloukal P, Ramlau R, et al. A randomized phase III trial comparing bexarotene/cisplatin/vinorelbine versus cisplatin/vinorelbine in chemotherapy-naive patients with advanced or metastatic non-small cell lung cancer. Proc Am Soc Clin Oncol 2005;23:7024.

39. Rosell R, Cobo M, Isla D, et al. ERCC1 mRNA-based randomized phase III trial of docetaxel doublets with cisplatin or gemcitabine in stage IV non-small cell lung cancer patients. Proc Am Soc Clin Oncol 2005;23:7002.

40. Shepherd F, Dancey J, Ramlau R, et al. Prospective randomized trial of docetaxel vs best supportive care in patients with non-small-cell lung cancer previously treated with platinum-based chemotherapy. J Clin Oncol 2000;18:2095-103.

41. Fossella F, DeVore R, Kerr R, et al. Randomized phase III trial of docetaxel versus vinorelbine or ifosfamide in patients with advanced non-small cell lung cancer previously treated with platinum-containing chemotherapy regimens. J Clin Oncol 2000;18:2354-62.

42. Hanna N, Shepherd F, Fossella F, et al. Randomized phase III trial of pemetrexed versus docetaxel in patients with non-small-cell lung cancer previously treated with chemotherapy. J Clin Oncol 2004; 22:1589-97.

43. Kris M, Natale R, Herbst R, et al. Efficacy of gefitinib, an inhibitor of the epidermal growth factor receptor tyrosine kinase, in symptomatic patients with non-small cell lung cancer: a randomized trial. JAMA 2003; 290:2149-58.

44. Fukuoka M, Yano S, Giaccone G, et al. Multi-institutional randomized phase II trial of gefitinib for previously treated patients with advanced non-small-cell lung cancer. J Clin Oncol 2003; 21:2237-46.

45. Pérez-Soler R, Chachoua A, Hammond L, et al. Determinants of tumor response and survival with erlotinib in patients with non-small-cell lung cancer. J Clin Oncol 2004; 22:3238-47.

46. Herbst RS, Johnson DH, Mininberg E, et al. Phase I/II trial evaluating the anti-vascular endothelial growth factor monoclonal antibody bevacizumab in combination with the HER-1/epidermal growth factor receptor tyrosine kinase inhibitor erlotinib for patients with recurrent non-small cell lung cancer. J Clin Oncol 2005;23:2544-55.

47. Shepherd F, Pereira J, Ciuleanu T, et al. A randomized placebo-controlled trial of erlotinib in patients with advanced non-small cell lung cancer (NSCLC) following failure of 1st line or 2nd line chemotherapy. A National Cancer Institute of Canada Clinical Trials Group trial. Proc Am Soc Clin Oncol 2004; 22:7022.

48. Thatcher N, Chang A, Parikh P, et al. ISEL: a phase III survival study comparing gefitinib (IRESSA) plus best supportive care (BSC) with placebo plus BSC, in patients with advanced non-small-cell lung cancer who had received one or two prior chemotherapy regimens. Lung Cancer 2005;49:S4.

49. Cufer T, Vrdoljak E. Results from a phase II, open-label study (SIGN) comparing gefitinib with docetaxel as second-line therapy in patients with advanced (stage IIIb or IV) non-small cell lung cancer. Proc Am Soc Clin Oncol 2005;23:7035.

50. Natale R, Bodkin D, Govindan R, et al. A comparison of the antitumor efficacy of ZD6474 and gefitinib (IRESSA) in patients with NSCLC: Results of a randomised doubleblind Phase II study. Lung Cancer 2005;49:S37.

51. Fanucchi M, Fossella F, Fidias P, et al. Bortezomib \pm docetaxel in previously treated patients with non-small cell lung 
cancer: A phase 2 study. Proc Am Soc Clin Oncol 2005;23:7034.

52. Lilenbaum R, Bonomi $\mathrm{P}$, Ansari R, et al. A phase II trial of cetuximab as therapy for recurrent non-small cell lung cancer: Final results. Proc Am Soc Clin Oncol 2005;23:7036.

53. Kim ES, Mauer AM, Tran HT, et al. A phase II study of cetuximab, an epidermal growth factor receptor (EGFR) blocking antibody, in combination with docetaxel in chemotherapy refractory/resistant patients with advanced nonsmall cell lung cancer: final report. Proc Am Soc Clin Oncol 2003;21:642a.

54. Gridelli C, Ardizzoni A, Le Chevalier T, et al. Treatment of advanced non-small cell lung cancer patients with ECOG performance status 2: results of an European Experts Panel. Ann Oncol 2004;15:419-26.

55. Effects of vinorelbine on quality of life and survival of elderly patients with advanced non-small-cell lung cancer. The Elderly Lung Cancer Vinorelbine Italian Study Group. J Natl Cancer Inst 1999; 91:66-72.

56. Gridelli, C, Perrone, F, Gallo, C, et al. Chemotherapy for Elderly Patients With Advanced Non-Small-Cell Lung Cancer: The Multicenter Italian Lung Cancer in the Elderly
Study (MILES) Phase III Randomized Trial. J Natl Cancer Inst 2003; 95:362-72.

57. Frasci G, Lorusso V, Panza N, et al. Gemcitabine plus vinorelbine versus vinorelbine alone in elderly patients with advanced non-small-cell lung cancer. J Clin Oncol 2000; 18:2529-36.

58. Takeda K, Kudoh S, Nakagawa K, et al. Randomized phase III study of docetaxel versus vinorelbine for elderly patients with advanced non-small cell lung cancer: Results of a West Japan Thoracic Oncology Group Trial (WJTOG9904). Proc Am Soc Clin Oncol 2005;23:7009.

59. Gridelli C, Aapro M, Ardizzoni A, et al. Treatment of advanced non-small cell lung cancer in the elderly: Results of an international expert panel. J Clin Oncol 2005;23:312537.

60. Stabile LP, Lyker JS, Gubish CT, et al. Combined targeting of the estrogen receptor and the epidermal growth factor receptor in non-small cell lung cancer shows enhanced antiproliferative effects. Cancer Res 2005;65:1459-70.

61. Dubey S, Siegfried J, Stabile L, et al. Combination therapy with gefitinib and fulvestrant for women with non-small cell lung cancer. Lung Cancer 2005;49:S87. 